\title{
Research Article \\ Social Effect Analysis of Intelligent Sports Based on Principal Component Analysis and Fuzzy Control
}

\author{
Xiaobo Chen $(\mathbb{D})$ \\ Sports Department, Shaanxi University of Science \& Technology, Shaanxi Xian 710021, China \\ Correspondence should be addressed to Xiaobo Chen; chenxiaobo@sust.edu.cn
}

Received 29 August 2021; Revised 29 September 2021; Accepted 15 October 2021; Published 31 October 2021

Academic Editor: Mu Zhou

Copyright (c) 2021 Xiaobo Chen. This is an open access article distributed under the Creative Commons Attribution License, which permits unrestricted use, distribution, and reproduction in any medium, provided the original work is properly cited.

\begin{abstract}
In order to explore the social effects of intelligent sports systems, this paper combines principal component analysis technology and fuzzy control technology to construct an intelligent sports social effect analysis system. The original fuzzy data is expressed linearly by structural elements, and the original fuzzy data matrix is divided into a main data matrix part and an error data matrix part. According to the principal component analysis method of fuzzy data represented by structural elements, this paper studies the principal component analysis method of interval data using the left end point matrix, right end point matrix, and midpoint matrix of interval data. In addition, this article uses principal component analysis and fuzzy control to study the response of the intelligent motion system to the masses and conducts experiments to analyze the social effects. It can be seen from experimental research that the intelligent sports system constructed in this article has a high degree of satisfaction of the masses, which proves that the intelligent sports have a certain social effect.
\end{abstract}

\section{Introduction}

With the development of wearable device technology, the mobile Internet has been further integrated into people's lives, and the use of smart devices in sports environments has become more frequent. As the wearable device with the largest market share in the current market, smart watches are of great significance to the study of their interface design in a sports environment, and to a certain extent, they have laid a design foundation for the development of the mobile Internet era. After smart watches entered the consumer market on a large scale in 2014, they were once considered to be smart personal terminal products that occupied a large number of markets after smart phones. However, by the second half of 2016, the survey showed that the growth rate of the smart watch market was far from reaching expectations, and the idle rates of purchased fitness trackers and smart watches were $30 \%$ and $29 \%$, respectively [1]. The survey shows that $49.5 \%$ of the global population wear watches, but most people who buy smart watches are still early adopters of technology products. Although it has a good market prospect, the design ideas of existing smart watches continue to be in smart phones. The screen size limitations, mobility, and context sensitivity of smart watches also pose new challenges for interface design.

With the progress of society and the improvement of the quality of life, more people begin to pay attention to their own health and begin to pursue a healthy lifestyle. The emergence of wearable smart devices provides another new way for people to pursue a healthy lifestyle.

Through these intelligent hardware that can be worn on the human body, it can connect and accommodate a variety of advanced technologies such as wireless communication, microsensing, GPS positioning, virtual reality, and biometric identification. Moreover, it can also integrate the Internet and big data platforms to search, analyze, share, and feedback on the human body anytime and anywhere. There are various types of wearable smart devices, such as smart watches, glasses, necklaces, or clothes. In recent years, wearable smart devices have been popularized and gradually accepted and respected by consumers. Smart watches can detect various data of the user during sports, such as time, distance, heart rate, calories, and other data, and are accepted by more and more young consumers. With the 
continuous improvement of technology, wearable smart devices have gradually shifted from focusing on hardware technology to more focusing on user experience. The improvement of interaction design has gradually increased the weight of smart watch design [2].

With the continuous popularization of smart sports equipment, it has caused a certain response in the society [3]. This article combines principal component analysis technology and fuzzy control technology to construct a social effect analysis system of intelligent sports and explores the response of intelligent sports equipment among the public.

\section{Related Work}

In recent years, the research and development of smart devices in the field of health monitoring at home and abroad have been very rapid, and the research on the detection of various physiological and physical parameters of the human body has been in-depth. The detected parameters mainly focus on body temperature, blood pressure, heart rate, pulse, fall, and so on. Along with the development of smart devices, the construction of sensor networks, big data, and cloud platforms is constantly deepening. The establishment of sports and health data centers is gradually changing the traditional methods of health monitoring and disease prevention and solving the problem of not having real-time performance when only relying on hospitals to diagnose diseases.

Smart watches have great application significance in personal life (for example, medical treatment), and it will become an important terminal in the science and technology ecosystem and penetrate into everyone's life [4]. Smart watches are used in family life and can be used to recognize human body abnormalities, recognize sleep conditions, and recognize gestures and other life situations [5]. Its core function is to monitor the health status at any time, and it is considered as a portable health assistant for humans [6]. In addition to life, smart watches also have better applications in work. Compared with smart phones, smart watches make tasks (for example, receiving notification information) more efficient and easier when completing some specific tasks [7].

When the smart watch is combined with other smart devices, some device interaction functions can be realized. The literature [8] integrated applications into smart watches to make the cross-device interaction between smart watches and smart phones a way to extend the terminal. The literature [9] further developed the joint interaction between smart watch and mobile phone, such as jointly completing touch input and screen expansion. The smart watch can also interact with the computer. When the user wears the watch for keyboard input, through the analysis of the smart watch sensor data, the characters typed by the test can be roughly estimated. When typing an English word with more than 6 characters, there are 10 possible options that can be identified. The loss of visible information causes a certain degree of difficulty in accurate recognition. The wearability of smart watches makes it possible for designers to use built-in sensor technology to capture motion and then perform data analysis and interact with other devices. The literature [10] first analyzed its necessity, and the literature [11] developed a gesture recognition system to solve the problems of highlevel interference, transmission data delay, and neglected actions. Based on action recognition, a smart watch-based App was developed, which mainly realizes functions such as controlling the opening and closing of the office door, obtaining information in the house, and simulating door knocking [12]. In addition, literature [13] developed another set of systems involving smart watches and mobile phones to interact with smart TVs. By capturing the movement of the hand wearing the smart watch in the air, the direction the user's hand that is pointing can be recognized. By comparing this method with the method using dedicated forearm sensors (Mypoint) and the method using camera capture technology (Vicon), it is found that the results of the smart watch solution can reach a relatively professional level [14]. In addition, by combining smart watches and smart phones to monitor the user's human activities, the interference of smart device notifications to workers can be reduced. By identifying whether the worker is working or resting, the system under study can adjust the notification push. The results of the research show that this method can significantly reduce the perceived workload of workers [15].

Information processing is the central part, mainly through the central processing unit to analyze and process the signals transmitted by the information detection unit, and schedule other hardware parts to jointly complete the predetermined tasks. This layer is the core layer of the entire smart clothing hardware part, with the function of intelligent decisionmaking. The pros and cons of information processing technology are directly related to the accuracy of signal processing, the complexity of calculation methods, and the size of power consumption [16]. The central processing unit is the core component of information processing, and the Bluetooth module transmits the data processed by the central processing unit to the external mobile terminal. Information feedback is to transmit the conclusion obtained by the analysis of the information processing unit to the monitoring platform terminal and feedback to the monitoring object in time, so that the monitoring individual or the monitoring center can react in real time. Existing transmission methods can be divided into two categories: wired and wireless transmission. The communication methods described in this article are mainly wireless transmission methods based on Bluetooth. The terminal application module mainly assists users in assessing their own physiological and physical conditions through the analysis of body data and gives users a good experience in a more flexible and intuitive way on the software interface [17].

The literature [18] concluded that wearable acceleration sensors can easily identify the gait performance of walking in daily environments. Compared with other devices, the acceleration sensor has the characteristics of portability and light cost and is considered to be an ideal choice for evaluating human movement and balance changes. Therefore, many researchers have specially designed sensors for acceleration data collection. Considering that the center of gravity of the body is close to the waist of the human body, most of these studies use sensors installed on the waist of the human body to measure gait characteristics [19]. In addition, because acceleration sensors are usually built into smart 
handheld devices, some researchers use such off-the-shelf products to measure body acceleration data during walking [20]. The results of the study show that the accuracy of gait measurement using an Android-based smartphone is equivalent to that of a customized three-axis accelerometer sensor [21], which expands new ideas for subsequent research.

2.1. The Main Matrix and Error Matrix of the Fuzzy Data Matrix. The classic principal component analysis method can directly process accurate data, but it cannot directly process fuzzy data represented by structural elements linearly. For this reason, this paper processes the fuzzy data matrix linearly represented by structural elements, divides the fuzzy data matrix into the main matrix part and the error matrix part, and uses the classical principal component analysis method to process the main matrix and the error matrix, respectively.

If there are $n$ observation samples, and $p$ indicators $X_{1}$, $X_{2}, \cdots, X_{p}$ are observed for each sample, the original sample data matrix is obtained as:

$$
X=\left[\begin{array}{cccc}
x_{11} & x_{12} & \cdots & x_{1 p} \\
x_{21} & x_{22} & \cdots & x_{2 p} \\
\vdots & \vdots & \ddots & \vdots \\
x_{n 1} & x_{n 2} & \cdots & x_{n p}
\end{array}\right]=\left(X_{1}, X_{2}, \cdots, X_{p}\right)
$$

In the formula, $X_{i}=\left(x_{1 i}, x_{2 i}, \cdots, x_{n i}\right), i=1,2, \cdots, p$.

For fuzzy principal component analysis, the object it studies is fuzzy data; that is, the data matrix under the condition that all samples is fuzzy data.

$$
\tilde{X}=\left[\begin{array}{cccc}
\tilde{x}_{11} & \tilde{x}_{12} & \cdots & \tilde{x}_{1 p} \\
\tilde{x}_{21} & \tilde{x}_{22} & \cdots & \tilde{x}_{2 p} \\
\vdots & \vdots & \ddots & \vdots \\
\tilde{x}_{n 1} & \tilde{x}_{n 2} & \cdots & \tilde{x}_{n p}
\end{array}\right]
$$

It is assumed that all fuzzy data can be linearly generated by the fuzzy structure element $E$, namely:

$$
\tilde{x}_{i j}=a_{i j}+b_{i j} E\left(b_{i j} \geq 0\right), i=1,2, \cdots, n ; j=1,2, \cdots, p .
$$

Then, there is

$$
\begin{aligned}
\tilde{X} & =\left[\begin{array}{cccc}
a_{11}+b_{11} E & a_{12}+b_{12} E & \cdots & a_{1 p}+b_{1 p} E \\
a_{21}+b_{21} E & a_{22}+b_{22} E & \cdots & a_{2 p}+b_{2 p} E \\
\vdots & \vdots & \ddots & \vdots \\
a_{n 1}+b_{n 1} E & a_{n 2}+b_{n 2} E & \cdots & a_{n p}+b_{n p} E
\end{array}\right] \\
& =\left(\tilde{X}_{1}, \tilde{X}_{2}, \cdots, \tilde{X}_{p}\right) .
\end{aligned}
$$

In the formula,

$$
\tilde{X}_{j}=\left(\begin{array}{c}
a_{1 j}+b_{1 j} E \\
a_{2 j}+b_{2 j} E \\
\vdots \\
a_{n j}+b_{n j} E
\end{array}\right)=A_{j}+B_{j} E, j=1,2, \cdots, p
$$

The fuzzy data matrix $\tilde{X}$ can be divided into two parts:

$$
\begin{aligned}
\tilde{X} & =\left[\begin{array}{cccc}
a_{11}+b_{11} E & a_{12}+b_{12} E & \cdots & a_{1 p}+b_{1 p} E \\
a_{21}+b_{21} E & a_{22}+b_{22} E & \cdots & a_{2 p}+b_{2 p} E \\
\vdots & \vdots & \ddots & \vdots \\
a_{n 1}+b_{n 1} E & a_{n 2}+b_{n 2} E & \cdots & a_{n p}+b_{n p} E
\end{array}\right] \\
& =\left[\begin{array}{cccc}
a_{11} & a_{12} & \cdots & a_{1 p} \\
a_{21} & a_{22} & \cdots & a_{2 p} \\
\vdots & \vdots & \ddots & \vdots \\
a_{n 1} & a_{n 2} & \cdots & a_{n p}
\end{array}\right]+\left[\begin{array}{cccc}
b_{11} & b_{12} & \cdots & b_{1 p} \\
b_{21} & b_{22} & \cdots & b_{2 p} \\
\vdots & \vdots & \ddots & \vdots \\
b_{n 1} & b_{n 2} & \cdots & b_{n p}
\end{array}\right] E \\
& =M+\mathrm{WE} .
\end{aligned}
$$

The matrix $M$ is called the main matrix of the fuzzy data matrix $\tilde{X}$, and $W$ is called the error matrix of the fuzzy data matrix $\tilde{X}$. The main matrix $M$ and the error matrix $W$ divide the fuzzy data into the main data part and the error data part.

In fuzzy analysis, interval number, as a special case of fuzzy number, represents a kind of uncertainty and has a wide range of applications in various fields. When conducting principal component analysis, if the data obtained is not deterministic data but interval data, how to obtain the principal components of interval data is the research focus.

If there are $n$ observation samples, and $P$ indicators $X_{1}$, $X_{2}, \cdots, X_{p}$ are observed for each sample, the original sample data matrix is obtained as:

$$
X=\left[\begin{array}{cccc}
x_{11} & x_{12} & \cdots & x_{1 p} \\
x_{21} & x_{22} & \cdots & x_{2 p} \\
\vdots & \vdots & \ddots & \vdots \\
x_{n 1} & x_{n 2} & \cdots & x_{n p}
\end{array}\right]=\left(X_{1}, X_{2}, \cdots, X_{p}\right)
$$

In the formula, $X_{i}=\left(x_{1 i}, x_{2 i}, \cdots, x_{n i}\right)^{T}, i=1,2, \cdots, p$.

For the principal component analysis method based on interval data, the object it studies is interval data; that is, all samples are interval data. Then, there is 


\begin{tabular}{|l|r|}
\hline \multicolumn{1}{|c|}{ Cloud security management } & $\begin{array}{c}\text { Cloud computing } \\
\text { management }\end{array}$ \\
\hline $\begin{array}{l}\text { Traditional applications and } \\
\text { services }\end{array}$ & $\begin{array}{r}\text { Big data applications and } \\
\text { services }\end{array}$ \\
\hline $\begin{array}{l}\text { Virtualization resource tools } \\
\text { Flexible resource plan } \\
\text { management }\end{array}$ & $\begin{array}{r}\text { Investigate analysis and extract } \\
\text { parallel algorithms }\end{array}$ \\
\hline Virtualization technology & Parallel computing \\
\hline \multicolumn{2}{|c|}{ Cloud computing resources and platforms } \\
\hline
\end{tabular}

FIgURE 1: Key contents of cloud computing.

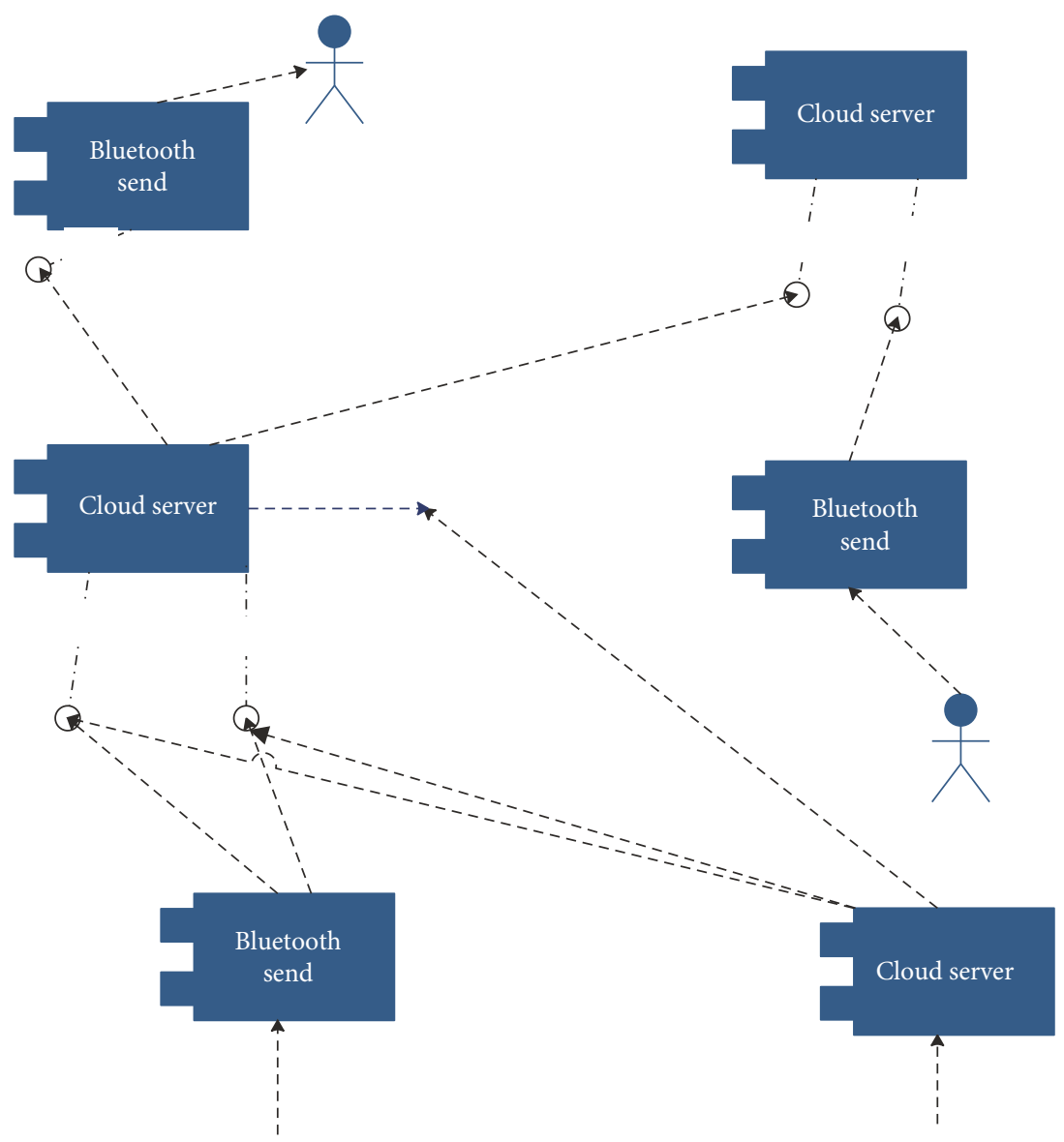

FIgURE 2: WebSocket protocol agreement. 


$$
\tilde{X}=\left[\begin{array}{cccc}
\tilde{x}_{11} & \tilde{x}_{12} & \cdots & \tilde{x}_{1 p} \\
\tilde{x}_{21} & \tilde{x}_{22} & \cdots & \tilde{x}_{2 p} \\
\vdots & \vdots & \ddots & \vdots \\
\tilde{x}_{n 1} & \tilde{x}_{n 2} & \cdots & \tilde{x}_{n p}
\end{array}\right] .
$$

In the formula, $\tilde{x}_{i j}=\left[a_{i j}, b_{i j}\right], i=1,2, \cdots, n ; j=1,2, \cdots, p$ is the interval number, so the obtained interval data sample matrix is

$$
\begin{aligned}
\tilde{X} & =\left[\begin{array}{cccc}
{\left[a_{11}, b_{11}\right]} & {\left[a_{12}, b_{12}\right]} & \cdots & {\left[a_{1 p}, b_{1 p}\right]} \\
{\left[a_{21}, b_{21}\right]} & {\left[a_{22}, b_{22}\right]} & \cdots & {\left[a_{2 p}, b_{2 p}\right]} \\
\vdots & \vdots & \ddots & \vdots \\
{\left[a_{n 1}, b_{n 1}\right]} & {\left[a_{n 2}, b_{n 2}\right]} & \cdots & {\left[a_{n p}, b_{n p}\right]}
\end{array}\right] \\
& =\left(\tilde{X}_{1}, \tilde{X}_{2}, \cdots, \tilde{X}_{p}\right) .
\end{aligned}
$$

In the formula,

$$
\tilde{X}_{j}=\left(\begin{array}{c}
{\left[a_{1 j}, b_{1 j}\right]} \\
{\left[a_{2 j}, b_{2 j}\right]} \\
\vdots \\
{\left[a_{n j}, b_{n j}\right]}
\end{array}\right), j=1,2, \cdots, p .
$$

We set $c_{i j}=\left(\left(a_{i j}+b_{i j}\right) / 2\right), i=1,2, \cdots, n ; j=1,2, \cdots, p$ is the midpoint of $a_{i j}$ and $b_{i j}$, and

$$
\begin{aligned}
& A=\left(a_{1 j}, a_{2 j}, \cdots, a_{n j}\right), j=1,2, \cdots, p, \\
& B=\left(b_{1 j}, b_{2 j}, \cdots, b_{n j}\right), j=1,2, \cdots, p, \\
& C=\left(c_{1 j}, c_{2 j}, \cdots, c_{n j}\right), j=1,2, \cdots, p .
\end{aligned}
$$

Then, $A, B$, and $C$ are three data matrices. $A$ is the matrix formed by the left end of the interval data, $B$ is the matrix formed by the right end of the interval data, and $C$ is the matrix formed by the midpoint of the interval data. Next, according to the classic principal component analysis method, the coefficient vectors $a_{j}^{0}, b_{j}^{0}, c_{j}^{0}, j=1,2, \cdots, m$ of the principal components of the three data matrices $A, B$, and $C$ are, respectively, obtained.

For the principal component analysis of fuzzy data, the original fuzzy data is divided into the main data part and the error data part. For the principal component analysis of interval data, the interval data matrix is divided into three data matrices $A, B$, and $C$, namely, the left end data matrix, the right end data matrix, and the midpoint data matrix. Then, the classical principal component analysis method is used to obtain the coefficient vector $a_{j}^{0}, b_{j}^{0}, c_{j}^{0}, j=1,2, \cdots$, $m$ of the three principal components, and the coefficient vector $k_{j}^{0}, j=1,2, \cdots, m$ of the principal component of the original interval data is obtained through the combination of the coefficient vector $a_{j}^{0}, b_{j}^{0}, c_{j}^{0}$ of the principal component.

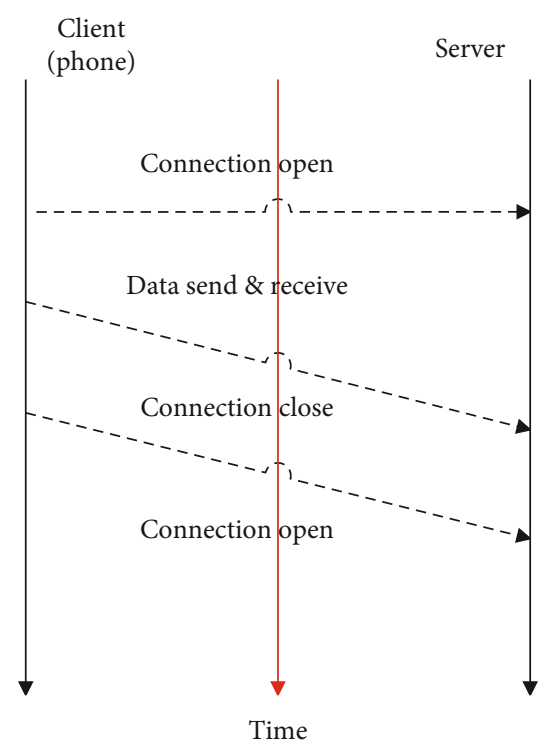

Figure 3: The communication process between the mobile phone and the server implemented by WebSocket.
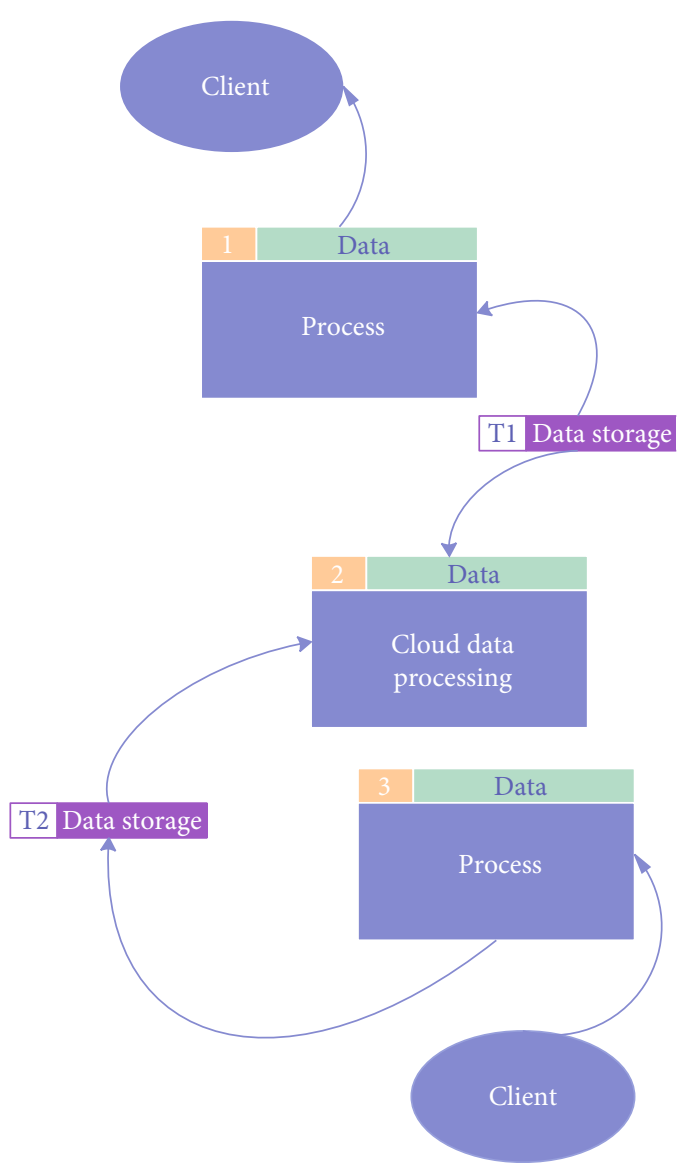

FIgURe 4: Data cloud storage model.

We assume that the coefficient vector of the principal component of the original interval data is $k_{j}^{0}$, the coefficient vector of the principal component of the left endpoint data 


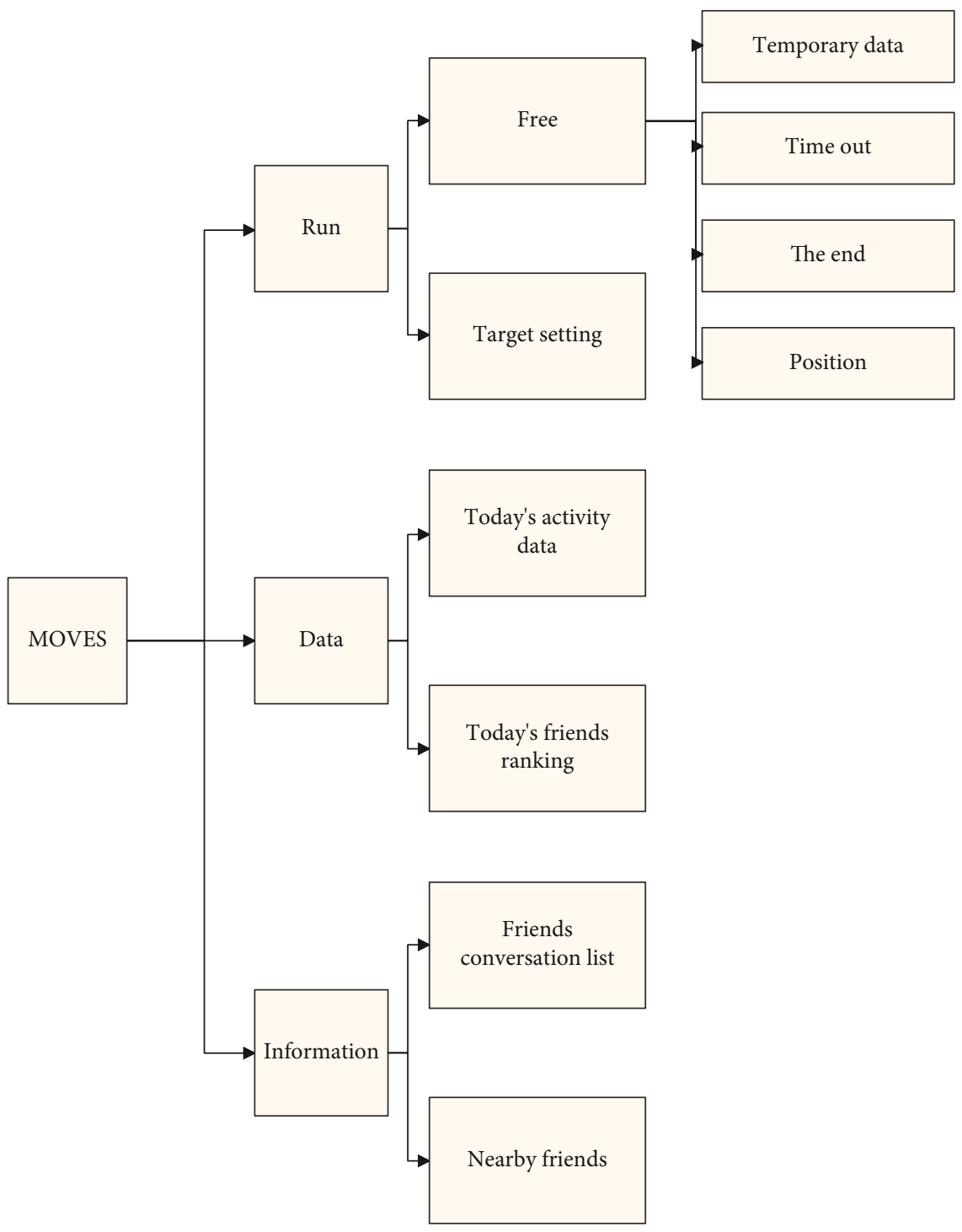

FIGURE 5: Information architecture diagram 1.

matrix $A$ is $a_{j}^{0}$, the coefficient vector of the principal component of the right endpoint data matrix $B$ is $b_{j}^{0}$, and the coefficient vector of the principal component of the midpoint data matrix $c$ is $c_{j}^{0}$. Then, the relationship between $k_{j}^{0}, a_{j}^{0}, b_{j}^{0}, c_{j}^{0}$ can be expressed as:

$$
\begin{aligned}
& k_{j}^{0}=\lambda a_{j}^{0}+\omega b_{j}^{0}+(1-\lambda-\omega) c_{j}^{0}, \\
& \lambda, \omega \in[0,1], \lambda+\omega<1, j=1,2, \cdots, m .
\end{aligned}
$$

After selecting the value of $\lambda, \omega, k_{j}^{0}, j=1,2, \cdots, m$ is obtained.

If $\left(k_{1}^{0}\right)^{2}+\left(k_{2}^{0}\right)^{2}+\cdots+\left(k_{m}^{0}\right)^{2}=1$, the principal component expression of interval data is

$$
\tilde{F}_{1}=k_{1}^{0} \tilde{X}_{1}+k_{2}^{0} \tilde{X}_{2}+\cdots+k_{m}^{0} \tilde{X}_{m}
$$

$$
\tilde{F}_{i}=k_{1}^{i} \tilde{X}_{1}+k_{2}^{i} \tilde{X}_{2}+\cdots+k_{m}^{i} \tilde{X}_{m}
$$

If $\left(k_{1}^{0}\right)^{2}+\left(k_{2}^{0}\right)^{2}+\cdots+\left(k_{m}^{0}\right)^{2} \neq 1$, in order to make $\left(k_{1}^{0}\right)^{2}$ $+\left(k_{2}^{0}\right)^{2}+\cdots+\left(k_{m}^{0}\right)^{2}=1$, we set

$$
\left(k_{1}^{0}\right)^{2}+\left(k_{2}^{0}\right)^{2}+\cdots+\left(k_{m}^{0}\right)^{2}=D,
$$

$$
\left(\frac{k_{1}^{0}}{\sqrt{D}}\right)^{2}+\left(\frac{k_{2}^{0}}{\sqrt{D}}\right)^{2}+\cdots+\left(\frac{k_{m}^{0}}{\sqrt{D}}\right)^{2}=1 .
$$

Then, the coefficient vector of the principal component analysis of the interval data is

$$
\beta_{1}^{0}=\frac{k_{1}^{0}}{\sqrt{D}}, \beta_{2}^{0}=\frac{k_{2}^{0}}{\sqrt{D}}, \cdots, \beta_{m}^{0}=\frac{k_{m}^{0}}{\sqrt{D}} .
$$


The principal component expression of interval data is

$$
\begin{gathered}
\tilde{F}_{1}=\beta_{1}^{0} \tilde{X}_{1}+\beta_{2}^{0} \tilde{X}_{2}+\cdots+\beta_{m}^{0} \tilde{X}_{m}, \\
\tilde{F}_{i}=\beta_{1}^{i} \tilde{X}_{1}+\beta_{2}^{i} \tilde{X}_{2}+\cdots+\beta_{m}^{i} \tilde{X}_{m} .
\end{gathered}
$$

We assume that the coefficient vector of the principal component of the original interval data is $k_{j}^{0}$, the coefficient vector of the principal component of the left endpoint data matrix $A$ is $a_{j}^{0}$, the coefficient vector of the principal component of the right endpoint data matrix $B$ is $b_{j}^{0}$, and the coefficient vector of the principal component of the midpoint data matrix $c$ is $c_{j}^{0}$. Then, the relationship between $k_{j}^{0}, a_{j}^{0}, b_{j}^{0}$ , $c_{j}^{0}$ can be expressed as:

$$
k_{j}^{0}=\frac{\left|a_{j}^{0}\right|}{\left|a_{j}^{0}\right|+\left|b_{j}^{0}\right|+\left|c_{j}^{0}\right|} a_{j}^{0}+\frac{\left|b_{j}^{0}\right|}{\left|a_{j}^{0}\right|+\left|b_{j}^{0}\right|+\left|c_{j}^{0}\right|} b_{j}^{0}+\frac{\left|c_{j}^{0}\right|}{\left|a_{j}^{0}\right|+\left|b_{j}^{0}\right|+\left|c_{j}^{0}\right|} c_{j}^{0} .
$$

According to the relevant steps, the principal component expression of the interval data is finally obtained.

\subsection{Intelligent Sports System Based on Principal Component} Analysis and Fuzzy Control. Before constructing the motion system, first analyze the system requirements.

Clear and visible at a glance: use black as the background of the smart device, and try not to use bright background colors in the background of the interface. On the one hand, it is to improve the readability of graphics and text. On the other hand, the black background can be seamlessly integrated with the black frame of the device, giving users no visual effect on the edge of the screen. At the same time, it will save power and extend the standby time. It is still necessary for small size equipment.

High efficiency: stable application, smooth animation, and convenient interaction are all high efficiency performance. The interaction of applications on smart devices should be in the simplest state, reducing the frequency of interface jumps, using very few interface completion functions to increase continuity, and minimize application interaction costs. Keep the text of the current page visually, set global functions, flatten the usage path, and reduce the user's memory burden.

Mobile design: the interface design of smart devices is different from that of mobile phones. It is more limited by a smaller visual space and more mobile usage scenarios. Therefore, to redesign, you cannot directly move the ready-made applications on the mobile phone, but the design style must be consistent with the style of the mobile application interface, including color tone, flattening, dynamic effects, or some special effects.

Color: try to choose bright and strong colors. Strong contrasting colors can increase the readability of pictures and text. Different colors can also be used to distinguish interactive areas. As a mobile device, the use environment of a smart device may include strong light or a dim environment.

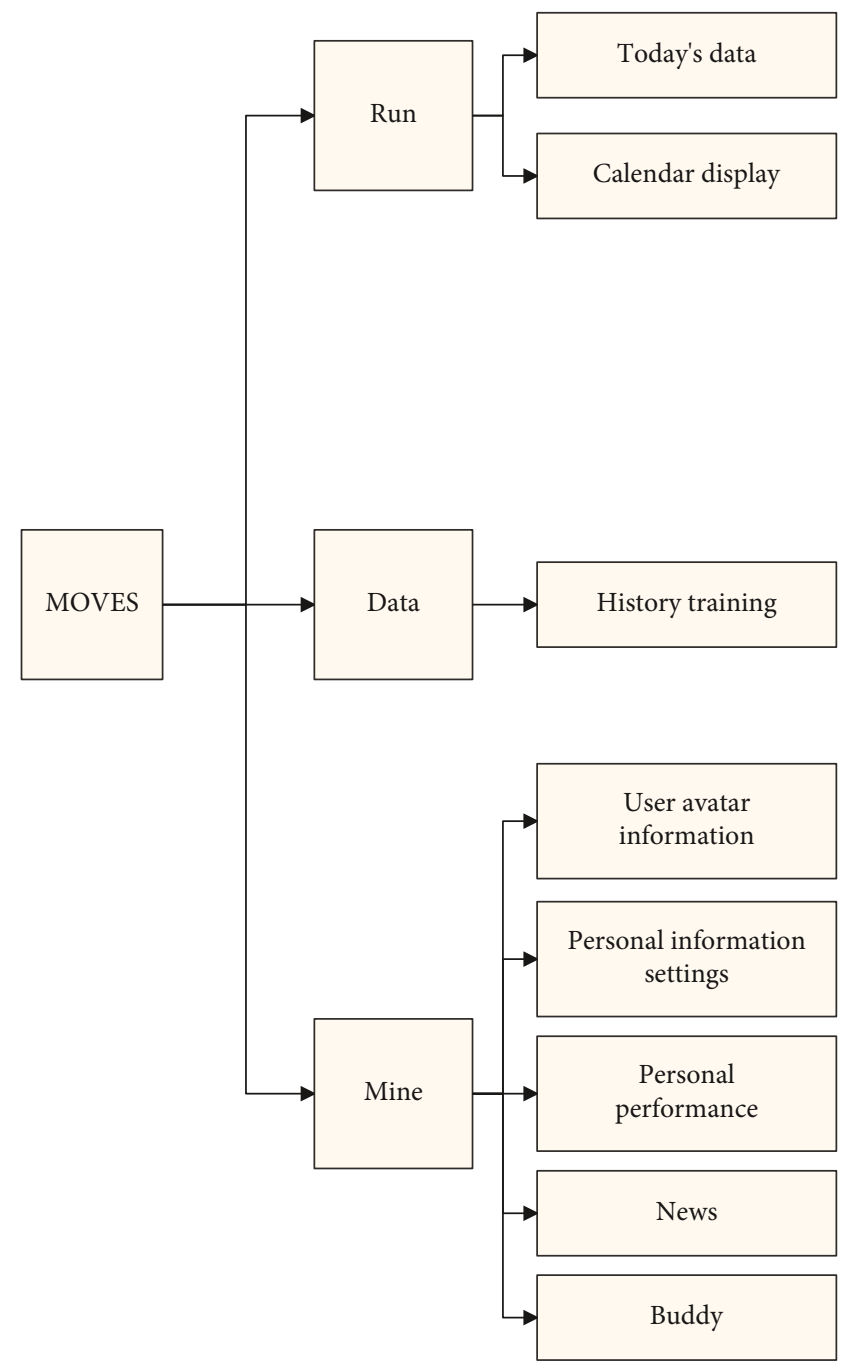

FIGURE 6: Information architecture diagram 2.

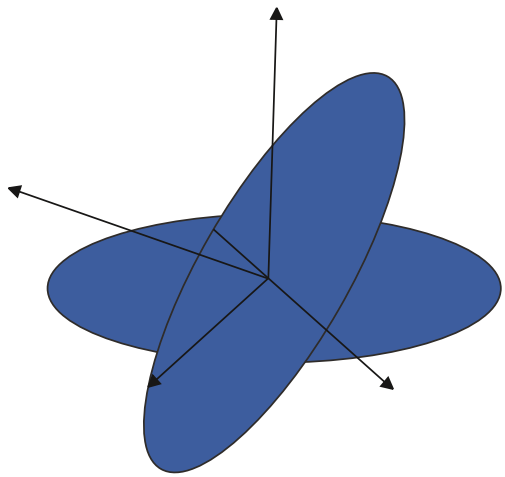

FIGURE 7: Euler space angle system.

Therefore, in order to ensure that pictures and text can be clearly seen in different environments, the choice of color is very important. To avoid low-saturation and softer colors, bright colors or high-saturation colors are better choices. Combining these colors with black or white fonts will achieve extremely high visual effects. 
TABLE 1: Statistical table of the health information collection effect of the intelligent sports system.

\begin{tabular}{|c|c|c|c|c|c|}
\hline Num & Health information collection effect & Num & Health information collection effect & Num & Health information collection effect \\
\hline 1 & 85.97 & 25 & 88.36 & 49 & 89.56 \\
\hline 2 & 88.60 & 26 & 80.08 & 50 & 90.39 \\
\hline 3 & 90.49 & 27 & 87.36 & 51 & 89.20 \\
\hline 4 & 86.48 & 28 & 88.42 & 52 & 90.55 \\
\hline 5 & 89.31 & 29 & 88.78 & 53 & 80.62 \\
\hline 6 & 86.53 & 30 & 81.25 & 54 & 82.44 \\
\hline 7 & 79.79 & 31 & 88.15 & 55 & 86.75 \\
\hline 8 & 84.88 & 32 & 89.94 & 56 & 85.41 \\
\hline 9 & 83.23 & 33 & 84.11 & 57 & 80.10 \\
\hline 10 & 80.20 & 34 & 82.81 & 58 & 89.70 \\
\hline 11 & 79.86 & 35 & 86.90 & 59 & 86.73 \\
\hline 12 & 82.24 & 36 & 81.95 & 60 & 85.43 \\
\hline 13 & 90.76 & 37 & 88.65 & 61 & 83.06 \\
\hline 14 & 85.03 & 38 & 83.73 & 62 & 87.45 \\
\hline 15 & 79.17 & 39 & 83.72 & 63 & 84.70 \\
\hline 16 & 83.28 & 40 & 80.94 & 64 & 83.50 \\
\hline 17 & 87.79 & 41 & 90.49 & 65 & 88.14 \\
\hline 18 & 84.49 & 42 & 81.14 & 66 & 82.38 \\
\hline 19 & 88.91 & 43 & 80.16 & 67 & 90.46 \\
\hline 20 & 83.30 & 44 & 90.46 & 68 & 81.99 \\
\hline 21 & 89.69 & 45 & 80.59 & 69 & 84.17 \\
\hline 22 & 84.23 & 46 & 79.44 & 70 & 84.27 \\
\hline 23 & 88.01 & 47 & 89.06 & 71 & 87.87 \\
\hline 24 & 79.85 & 48 & 79.28 & 72 & 79.65 \\
\hline
\end{tabular}

The establishment of big data and cloud computing platforms relies on some basic technologies, including the establishment of sensor networks and data centers. For big data, a flexible hardware infrastructure is a crucial part of realizing data storage. These hardware infrastructures include a large amount of flexible and shared information and communication technology resources. These information and communication technologies can be expanded and contracted horizontally or vertically and dynamically configured according to different application requirements. The advantages of cloud computing over the years have changed the way people obtain and use hardware infrastructure and software services. This view is mainly derived from the evolution of public computing and virtualized infrastructure. The key components of cloud computing are described in Figure 1.

For big data, the data center is not only the center of data storage but also needs to bear more responsibilities such as obtaining data, managing data, and mining the potential value and functions of data. The main focus of the data center is on the "data" rather than the "center." A data center has a large amount of data and managers and manages the data according to its core goals and development path, which is more valuable than having a good website and resources. The development of big data brings more development opportunities to the establishment of future data centers and at the same time faces greater challenges.
Big data requires strong back-end support from the data center. Big data places more stringent requirements on data centers in terms of storage capacity, processing capacity, and network transmission capacity. Only when a highly efficient, stable, secure, and scalable data center is established can the application of big data be ensured. The growing application of big data has also accelerated the reform of data centers. Many big data applications have developed their own unique structures and directly promoted the development of storage, network, and computing technologies related to data centers. With the continuous growth of structured and semistructured data forms, the means of analyzing data become more diversified, and data centers should strengthen their data processing and computing capabilities. Big data has given the data center more functions. For big data, the data center should not only focus on the establishment of hardware infrastructure but also strengthen software capabilities. This capability includes the acquisition, processing, management, analysis, and application of big data. The establishment of a monitoring center that sports and body temperature monitoring smart clothing will rely on in the future will place more emphasis on analysis and application, which will be an innovation in monitoring methods.

WebSocket is a technology invented to connect the client and the server. The WebSocket protocol is essentially a TCPbased protocol. It first initiates a special http request through the HTTP/HTTPS protocol for handshake and then creates 


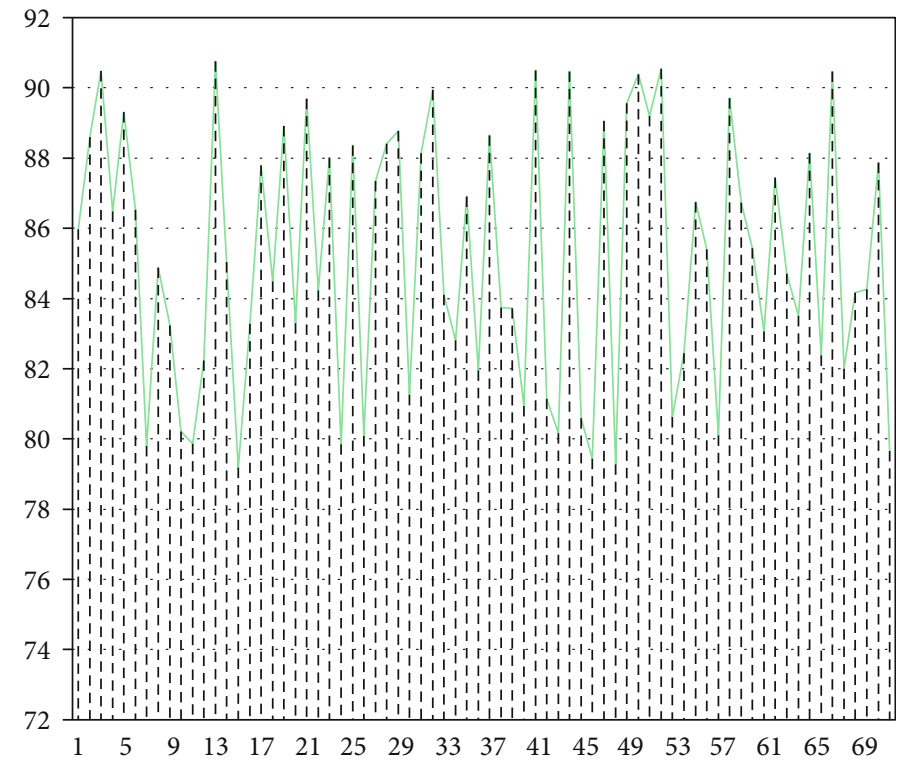

FIGURE 8: Statistical diagram of the health information collection effect of the intelligent sports system.

TABLE 2: Statistical table of crowd satisfaction of intelligent sports system.

\begin{tabular}{|c|c|c|c|c|c|}
\hline Num & Crowd satisfaction & Num & Crowd satisfaction & Num & Crowd satisfaction \\
\hline 1 & 92.20 & 25 & 94.16 & 49 & 82.03 \\
\hline 2 & 88.89 & 26 & 92.15 & 50 & 92.09 \\
\hline 3 & 92.87 & 27 & 92.12 & 51 & 88.88 \\
\hline 4 & 85.43 & 28 & 93.32 & 52 & 91.05 \\
\hline 5 & 94.67 & 29 & 91.54 & 53 & 83.30 \\
\hline 6 & 89.80 & 30 & 90.92 & 54 & 87.24 \\
\hline 7 & 85.64 & 31 & 94.07 & 55 & 84.45 \\
\hline 8 & 82.18 & 32 & 93.03 & 56 & 91.89 \\
\hline 9 & 92.92 & 33 & 94.45 & 57 & 91.38 \\
\hline 10 & 85.67 & 34 & 86.51 & 58 & 87.85 \\
\hline 11 & 83.17 & 35 & 83.88 & 59 & 82.65 \\
\hline 12 & 85.35 & 36 & 87.62 & 60 & 91.34 \\
\hline 13 & 85.35 & 37 & 90.53 & 61 & 82.85 \\
\hline 14 & 83.47 & 38 & 86.43 & 62 & 91.20 \\
\hline 15 & 86.69 & 39 & 83.26 & 63 & 90.59 \\
\hline 16 & 91.87 & 40 & 90.97 & 64 & 92.22 \\
\hline 17 & 85.29 & 41 & 89.80 & 65 & 85.98 \\
\hline 18 & 86.36 & 42 & 85.30 & 66 & 86.92 \\
\hline 19 & 83.13 & 43 & 92.51 & 67 & 82.04 \\
\hline 20 & 82.63 & 44 & 82.65 & 68 & 88.98 \\
\hline 21 & 86.63 & 45 & 92.15 & 69 & 89.01 \\
\hline 22 & 83.77 & 46 & 90.82 & 70 & 93.48 \\
\hline 23 & 88.82 & 47 & 88.54 & 71 & 84.53 \\
\hline 24 & 88.34 & 48 & 85.21 & 72 & 88.14 \\
\hline
\end{tabular}

a stable TCP connection to facilitate data exchange. After the connection is established, the server and the client can carry out real-time communication services through this TCP connection, and this communication mode is two-way communication, as shown in Figure 2. Figure 3 shows the communication process between the mobile phone and the server through WebSocket. 


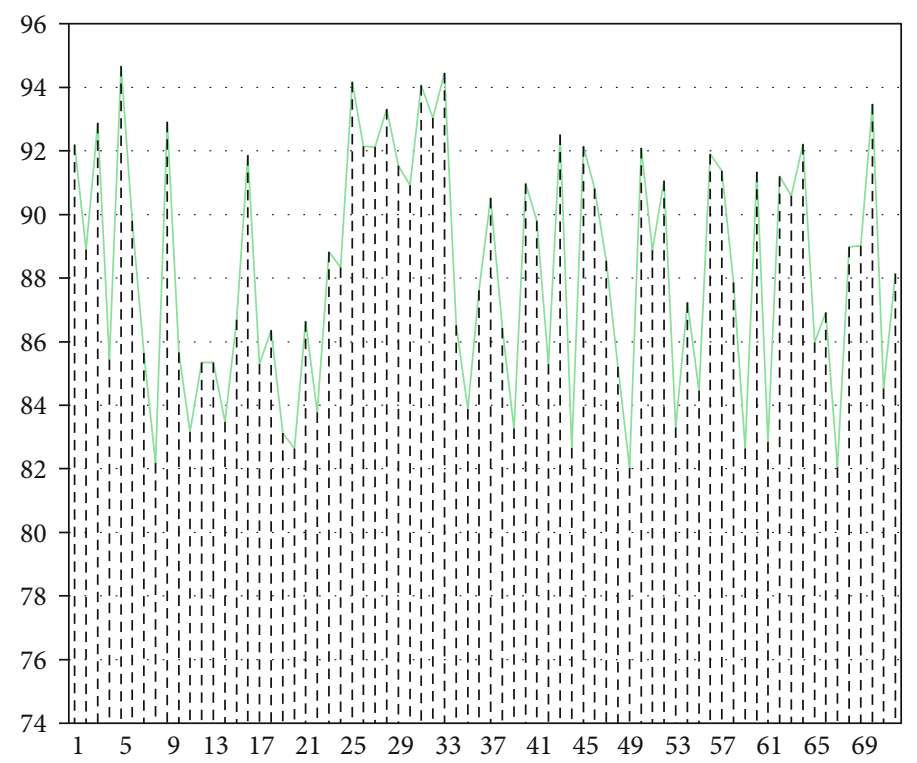

FIGURE 9: Statistical diagram of crowd satisfaction of intelligent sports system.

Because Mongo has a powerful query language and its syntax is a bit similar to object-oriented, most functions such as single-table query of relational database can be realized by it, and the form index function can be easily realized. The data cloud storage model is shown in Figure 4.

The system information architecture diagram constructed in this paper is shown in Figures 5 and 6.

This article draws on Euler's angle system in the aspect of system induction. As shown in Figure 7, an Euler space angle system is used to determine the position of a fixedpoint rotating rigid body with a set of three independent angle parameters, consisting of nutation angle $\alpha$, precession angle (i.e., precession angle) $\beta$, and rotation angle $\gamma$. Relative to the spatial reference system (blue), the reference system (red) fixed to the rigid body undergoes spatial rotation. At this time, the angle between the $z$ axis and the $Z$ axis is $\alpha$, the angle between the $x$ axis and the $N$ axis (the intersection line of the $x y$ plane and the $X Y$ plane) is $\beta$, and the angle between the $X$ axis and the $N$ axis is $\gamma$.

2.3. Analysis of Social Effects of Intelligent Sports Systems Based on Principal Component Analysis and Fuzzy Control. This article analyzes the effects of the intelligent sports system in the society and combines the intelligent sports system constructed in this article to study the reaction of the intelligent system in the masses through experimental analysis. On the basis of the above analysis, this article designs experiments to first verify the system's function of collecting people's health information through grouping experiments. The data obtained is shown in Table 1 and Figure 8.

From the above analysis results, the system constructed in this paper basically meets the basic requirements of sports health information collection. On this basis, this article uses the satisfaction survey method to study the response of the intelligent sports system in the masses. The results are shown in Table 2 and Figure 9.
From the above statistical results, the intelligent sports system constructed in this article has a good response in the masses. It can be seen that the intelligent sports system has a certain social effect and will definitely play a more important role in the health management of the masses in the future.

\section{Conclusion}

This article makes a systematic review of the current research status of smart sports equipment and analyzes the direction and focus of the next research. This paper develops the research content of the predecessors through experimental methods and continuously refines the information volume and information density presentation based on the previous research on the model structure, system display form, and layout. Moreover, this article conducts an indepth study on the interface operation and gesture operation of using smart sports equipment in the sports state.

The research purpose of this article is mainly to conduct research on the reaction of the intelligent motion system in the social masses through principal component analysis and fuzzy control. Moreover, this article combines the intelligent motion system constructed in this article to analyze and study the response of the intelligent sports system in the masses through experiments. From the research results, it can be seen that the intelligent sports system constructed in this article has a good response among the masses, and it can be seen that the intelligent sports have a certain social effect.

\section{Data Availability}

The labeled dataset used to support the findings of this study are available from the corresponding author upon request. 


\section{Conflicts of Interest}

The author declares no competing interests.

\section{Acknowledgments}

This study is sponsored by the Project of special scientific research plan of Shaanxi Provincial Department of Education, development and research of college students' fitness prescription system based on Wed database management system (No. 11jk0435).

\section{References}

[1] C. A. Emery and K. Pasanen, "Current trends in sport injury prevention," Best Practice \& Research Clinical Rheumatology, vol. 33, no. 1, pp. 3-15, 2019.

[2] V. M. Shulyatyev and M. A. Bulavina, "Sport interview: strategy, design and content," Theory and Practice of Physical Culture, vol. 9, pp. 18-18, 2019.

[3] L. J. Ferguson, K. T. Carlson, and D. Rogers, "Moving towards reconciliation through sport: Sharing our process of exploring team Saskatchewan experiences at the North American Indigenous Games," Journal of Exercise, Movement, and Sport (SCAPPS refereed abstracts repository), vol. 51, no. 1, pp. 9999, 2019.

[4] K. Kimasi, V. Shojaei, and M. R. Boroumand, "Investigation of safety conditions at gymnasia in different organizations," Journal of Humanities Insights, vol. 3, no. 2, pp. 70-74, 2019.

[5] K. Reinhart and B. Wichmann, "The TuS Fortschritt Magdeburg-Neustadt (soccer section) in the GDR-an example of amateur socialist sport," Soccer \& Society, vol. 21, no. 4, pp. 408-420, 2020.

[6] C. Abanazir, "E-sport and the EU: the view from the English Bridge Union," The International Sports Law Journal, vol. 18, no. 3-4, pp. 102-113, 2019.

[7] A. Gerke, K. Babiak, G. Dickson, and M. Desbordes, "Developmental processes and motivations for linkages in cross-sectoral sport clusters," Sport Management Review, vol. 21, no. 2, pp. 133-146, 2018.

[8] A. I. Pogrebnoy and I. O. Komlev, "Sport institutions reporting to Ministry of Sport of Russian Federation: intellectual property, invention activity, patenting and legal consulting service analysis," Theory and Practice of Physical Culture, vol. 2, 2018.

[9] D. C. Ilies, R. Buhas, and M. Ilies, "Sport activities and leisure in Nature 2000 protected area-Red Valley, Romania," Journal of Environmental Protection and Ecology, vol. 19, no. 1, pp. 367-372, 2018.

[10] A. I. Kondrukh, "Practical shooting sport in Russian sport system: essential specifications and features," Theory and Practice of Physical Culture, vol. 5, pp. 27-27, 2017.

[11] R. Giulianotti and D. Numerato, "Global sport and consumer culture: an introduction," Journal of Consumer Culture, vol. 18, no. 2, pp. 229-240, 2018.

[12] A. G. Gurinovich and G. V. Petrova, "Key priorities of physical education and sport sector budgeting laws and regulations in the Russian Federation," Theory and Practice of Physical Culture, vol. 4, pp. 34-34, 2019.
[13] M. Mountjoy, A. Costa, R. Budgett et al., "Health promotion through sport: international sport federations' priorities, actions and opportunities," British Journal of Sports Medicine, vol. 52, no. 1, pp. 54-60, 2018.

[14] J. J. Pulido, D. Sánchez-Oliva, P. A. Sánchez-Miguel, D. Amado, and T. García-Calvo, "Sport commitment in young soccer players: a self-determination perspective," International Journal of Sports Science \& Coaching, vol. 13, no. 2, pp. 243252, 2018.

[15] J. Cristiani, J. C. Bressan, and B. L. Pérez, "Clubs sociodeportivos en un municipio brasileño: espacio, equipos y contenidos [Sport clubs in Brazil: facilities, equipment and content in][Clubes socio-esportivos em município brasileiro: Espaço, equipamentos e conteúdos]," E-balonmano. com: Revista de Ciencias del Deporte, vol. 13, no. 2, pp. 105-112, 2017.

[16] E. Happ, M. Schnitzer, and M. Peters, "Sport-specific factors affecting location decisions in business to business sport manufacturing companies: a qualitative study in the Alps," International Journal of Sport Management and Marketing, vol. 21, no. 1/2, pp. 21-48, 2021.

[17] M. Castro-Sánchez, F. Zurita-Ortega, and R. Chacón-Cuberos, "Motivation towards sport based on sociodemographic variables in university students from Granada," Journal of Sport and Health Research, vol. 11, no. 1, pp. 55-68, 2019.

[18] S. M. Hadlow, D. Panchuk, D. L. Mann, M. R. Portus, and B. Abernethy, "Modified perceptual training in sport: a new classification framework," Journal of Science and Medicine in Sport, vol. 21, no. 9, pp. 950-958, 2018.

[19] J. H. Du Plessis and M. Berteanu, "The importance of prosthetic devices in sport activities for Romanian amputees who compete in Paralympic competitions," Medicina Sportiva: Journal of Romanian Sports Medicine Society, vol. 16, no. 1, pp. 3197-3204, 2020.

[20] M. Stylianou, A. Hogan, and E. Enright, "Youth sport policy: the enactment and possibilities of 'soft policy' in schools," Sport, Education and Society, vol. 24, no. 2, pp. 182-194, 2019.

[21] S. A. Richmond, A. Donaldson, A. Macpherson et al., "Facilitators and barriers to the implementation of iSPRINT: a sport injury prevention program in junior high schools," Clinical Journal of Sport Medicine, vol. 30, no. 3, pp. 231-238, 2020. 